\title{
$\$$ Research Square

\section{Characterization of Klebsiella pneumoniae isolated from patients suspected of pulmonary or bubonic plague during the Madagascar epidemic in 2017}

\author{
Andriniaina Rakotondra ( $\sim$ aina@pasteur.mg ) \\ Experimental bacteriology unit, Institut Pasteur Madagascar, Antananarivo
}

\section{Lova Andrianonimiadana}

Experimental bacteriology unit, Institut Pasteur Madagascar, Antananarivo

\section{Soloandry Rahajandraibe}

Plague unit, Institut Pasteur Madagascar, Antananarivo

\section{Solohery Razafimahatratra}

Experimental bacteriology unit, Institut Pasteur Madagascar, Antananarivo

\section{Voahangy Andrianaivoarimanana}

Plague unit, Institut Pasteur Madagascar, Antananarivo

\section{Soanandrasana Rahelinirina}

Plague unit, Institut Pasteur Madagascar, Antananarivo

\section{Tania Crucitti}

Experimental bacteriology unit, Institut Pasteur Madagascar, Antananarivo

\section{Sylvain Brisse}

Biodiversity and Epidemiology of Bacterial Pathogens, Institut Pasteur, Paris

\section{Victor Jeannoda}

Mention Biodiversité et Santé, Sciences Faculty, University of Antananarivo

\section{Minoarisoa Rajerison}

Plague unit, Institut Pasteur Madagascar, Antananarivo

Jean-Marc Collard

Experimental Bacteriology Laboratory, Center for Microbes, Development and Health (CMDH), Institut Pasteur of Shanghai/Chinese Academy of Sciences

\section{Research Article}

Keywords: Klebsiella pneumoniae, pneumopathy, bubo, community-acquired pneumonia, virulent clone.

Posted Date: May 3rd, 2021

DOl: https://doi.org/10.21203/rs.3.rs-449539/v1 
License: (c) (i) This work is licensed under a Creative Commons Attribution 4.0 International License. Read Full License 
1 Characterization of Klebs ie lla pneumoniae is olated from patients suspected of

2 pulmonary or bubonic plague during the Madagas car epide mic in 2017

3

4 Andriniaina Rakotondras oa ${ }^{1 *}$ Lova Maminirina Andrianonimiadana, ${ }^{1}$ Soloandry

5 Rahajandraibe, ${ }^{2}$ Solohery Razafimahatratra, ${ }^{1}$ Voahangy Andrianaivoarimanana, ${ }^{2}$

6 Soanandrasana Rahelinirina, ${ }^{2}$ Tania Crucitti, ${ }^{1}$ Sylvain Bris se, ${ }^{3}$ Victor Jeannoda, ${ }^{4}$

7 Minoaris oa Raje ris on, ${ }^{2 \#}$, Jean-Marc Collard. ${ }^{1 \# a}$

8

$9{ }^{1}$ Experimental bacteriology unit, Institut Pasteur Madagascar, Antananarivo, Madagascar;

$10 \quad{ }^{2}$ Plague unit, Institut Pasteur Madagascar, Antananarivo, Madagascar;

$11{ }^{3}$ Biodiversity and Epidemiology of Bacterial Pathogens, Institut Pasteur, Paris, France;

${ }^{4}$ Mention Biodiversité et Santé, Sciences Faculty, University of Antananarivo, Madagascar

$13{ }^{a}$ Current address: Experimental Bacteriology Laboratory, Center for Microbes, Development 14 and Health $(\mathrm{CMDH})$, Institut Pasteur of Shanghai/Chinese Academy of Sciences, People's 15 Republic of China

*Correspondence author: aina@pasteur.mg; Tel.: +261349818532

\# These authors contributed equally to this work 


\section{Abs tract}

Klebsiella pneumoniae can lead to a wide range of diseases including pneumonia, bloodstream and urinary tract infections. During a short period of a plague epidemic in October 2017 in Madagascar, 12 K. pne umoniae isolates were identified in ten sputum and two buboes aspirate samples. These isolates were from 12 patients suspected of plague, without epidemiological relationships, but were negative for Yersinia pestis in culture. Data were collected from the plague national surveillance system. The isolates were characterized by antimicrobial susceptibility testing and whole-genome sequencing. Real-time PCR was performed to confirm the presence of $K$. pneumoniae DNA in buboes. All isolates were identified as $K$. pneumoniae sensu stricto. Five isolates were extended-spectrum Blactamases producers; eight different sequence types were identified. Five isolates belonged to known hypervirulent sequence types. Our results demonstrate community-acquired pneumonia caused by K. pneumoniae isolates in patients suspected of plague, showing that plague epidemics can hide other etiologies.

Keywords : Klebs ie lla pne umoniae, pneumopathy, bubo, community-acquired pneumonia, virulent clone. 


\section{Background}

Between August 1 and November 26, 2017; a total of 2.414 clinically suspected plague cases were reported to the Central Laboratory for Plague (CLP) at the Institut Pasteur de Madagascar, including 1.878 (78\%) pulmonary plague (PP), 395 (16\%) bubonic plague (BP), one $(<1 \%)$ septicaemia and $140(6 \%)$ cases with unspecified clinical form ${ }^{1}$. This predominantly urban plague epidemic was characterised by a large volume of notifications in two major urban areas (Antananarivo and Toamasina) and by an unusually high proportion of pneumonic forms. According to the 2006 WHO standard plague case definitions and using the results of three types of diagnostic tests assessed (rapid F1-antigen diagnostic test, (RDT), molecular amplification method, and culture $)^{2}, 386 / 1.878(21 \%)$ were probable and $32 / 1.878(2 \%)$ were confirmed cases among the notified PP cases. The magnitude of this pneumonic plague outbreak is likely to have been smaller that suggested by notified suspected cases $^{1}$; and its severity indicated by the case fatality rate among confirmed plus probable (about 9\%) was substantially lower than observed the last previous 18 years $(25 \%)^{3}$. Over-reporting of PP cases due to limited clinical experience two most affected areas, and the difficulty to clinically diagnose PP through respiratory signs was speculated. The clinical diagnosis of pneumonic plague from polymicrobial sputum associated with other potential causes of pneumonia remains a challenge because the isolation of Yersinia pestis (Y.pestis) is more complicated compared to other bacteria.

Klebsiella pneumoniae (K. pneumoniae) is a Gram-negative bacterium naturally resistant to amoxicillin and carbenicillin. Klebsiella pneumoniae complex members comprise 7 phylogroups (Kp1 to Kp7) with $K$. pneumoniae sensu stricto, K. quasipneumoniae subsp. quasipneumoniae, K. quasipneumoniae subsp. similipneumoniae, K. variicola subsp. variicola, $K$. variicola subsp. tropica, $K$. quasivariicola, and $K$. africana, respectively ${ }^{4}$. It has become an important MDR pathogen of the last decade with multiple resistance determinants, mostly for aminoglycosides, cephalosporins and carbapenems ${ }^{5}$. It has been 
isolated from hospital-acquired infections including pneumonia, bloodstream infection, urinary tract infection, and community acquired infections such as hepatic abscess and meningitis and pneumonia. The capsule is an important virulence factor that protects $K$. pne umoniae from phagocytosis, with over 79 defined capsular serotypes. Isolates with $\mathrm{K} 1$ and K2 capsular serotypes are associated with virulent infections. However, not all K2 capsular isolates are virulent. In this regard, virulence was confirmed in murine models ${ }^{6}$. Virulence factors associated with hypervirulent Kle bs ie lla infections include siderophores, virulence plasmid encoding aerobactin, salmochelin, yersiniabactin, and the hypermucoviscosity factor $m p A / m p A 2$ gene $^{7-12}$.

Here we report on the characterization of a series of $12 \mathrm{~K}$. pneumoniae isolated from the sputum and bubo aspirates from clinically suspected plague patients during the plague outbreak in Madagascar, 2017.

\section{Mate rial and methods}

\section{Patients and bacterial is olates}

Patients with confirmed $K$. pneumoniae, isolated during nine days of the plague epidemic (from the $6^{\text {th }}$ of October 2017 till the $14^{\text {th }}$ of October 2017), were included in this sub-study. Epidemiological, clinical and lab data of patients were extracted from the plague national surveillance system database of Institut Pasteur de Madagascar between August $1^{\text {st }}$ and November $26^{\text {th }}$ in $2017^{1}$. Y. pestis was isolated from biological samples (bubo aspirates for bubonic plague, sputum for pneumonic plague) by direct culture on Yersinia selective Cefsulodin-Irgasan-Novobiocin (CIN) agar medium (Oxoid Ltd., United Kingdom). All methods were carried out according to the 2006 WHO recommendations ${ }^{13}$. Culture incubation was done at $26-28$ ?C for $48 \mathrm{~h}$ or longer as $Y$. pestis grows slower than other bacteria. Colonies obtained within $24 \mathrm{~h}$ on $\mathrm{CIN}$ medium and which did not have $Y$. pestis morphology were identified on MALDI-TOF MS (Biotyper version 3.3, Bruker Daltonics, 
Champs-sur-Marne, France). Colonies identified as K. pne umoniae were further purified on Simmons Citrate Agar Inositol (SCAI) medium ${ }^{14}$.

\section{Phenotype detection}

The hypermucoviscosity phenotype of the K. pneumoniae isolates was determined using the string test, in which a standard bacteriological loop is used to stretch a mucoviscous string from each colony cultured on SCAI medium. The formation of a viscous string $>5 \mathrm{~mm}$ in length was regarded as a positive test result ${ }^{12}$.

\section{Bacte rial susceptibility tes ting}

Antibiotic resistance profiles were determined by the standard disc diffusion method according to CASFM-EUCAST V2-0-May2017 guidelines and using breakpoints for Enterobacteriaceae (http://www.sfm-microbiologie.org). Isolates were tested against 17 commonly used antimicrobial agents, namely amoxicillin, amoxicillin-clavulanate, piperacillin/tazobactam, cefalotin, cefoxitin, cefotaxime, ceftazidime, cefepime, aztreonam, imipenem, ertapenem, tobramycin, gentamicin, nalidixic acid, ciprofloxacin, trimethoprimsulfamethoxazole and tetracycline. In addition, extended spectrum ß-lactamase (ESBL) production was tested using the standard double disc synergy test.

\section{Genome sequencing and analys is}

Genomic DNA of the K. pne umoniae isolates was extracted using DNeasy Blood \& Tissue kit (Qiagen, Germany) and was subjected to whole genome sequencing. Genomic libraries were constructed using the Nextera XT DNA library preparation kit with dual indexing (Illumina, San Diego, USA). The libraries were sequenced on an Illumina NextSeq-500. Genome assembly was performed de novo using Spades Genome Assembler (Version 3.10.0). Genome analyses for core genome MLST (cgMLST) on 632 core gene were performed, 
sequence types (ST), virulence genes, and capsular serotypes (K-types) were assigned using the Klebsiella pneumoniae database hosted through the BIGSdb web application of the Institut Pasteur in Paris (https://bigsdb.pasteur.fr/klebsiella ). Antimicrobial resistance genes were identified from genome sequences using the Resfinder (version 3.2). Plasmid replicon types were determined by using the Plasmidfinder (version 2.0) tool at https://genomicepidemiology.org/. RAxML and parsnp were used to draw the tree. The Gubbins software tool was used to remove the single nucleotide polymorphisms (SNPs) on recombined regions and to create a phylogenetic tree using Maximum Likelihood. The tree was subsequently annotated with iTOL.

Buboes K. pneumoniae screening by real-time PCR

We performed a real-time PCR targeting the zur-khe intergenic region ${ }^{15}$ on the bubo samples in order to confirm the presence of $K$. pneumoniae DNA in the bubo and to exclude any technical contamination during culture. Bacterial DNA was extracted from the bubo samples using DNeasy Blood \& Tissue kit (Qiagen, Germany). The real-time PCR assay was performed as previously described with the difference that we used $10 \mu$ of Sso Advanced universal SYBR Green Supermix (Bio-Rad, USA) ${ }^{15}$. Amplifications were performed using the CFX-96 (BIO-RAD, USA) platform. The positive controls consisted of DNA from $K$. pneumoniae UAA2239 and UAA2016 which are reference strains from the National Reference Center for Antibiotics from the Institut Pasteur in Paris, the negative control was 138 plain molecular grade water.

\section{Ethics s tatement}

141 No additional ethical clearance was requested because the analysis was carried out within the framework of the national surveillance system. No additional data was collected. 
All patients provided oral consent and voluntarily agreed for sampling for diagnostic purposes.

\section{Nucleotide sequence acces $s$ ion numbers}

WGS data have been deposited at the National Center for Biotechnology Information (NCBI) under BioProject PRJNA565154.

\section{Results}

Case presentation and Klebs ie lla pneumoniae antimic robial susceptibility

K. pne um oniae was isolated from 12 clinical samples out of 496 collected between 06 and 14 October 2017 in Antananarivo ( $\mathrm{N}=362)$ and Toamasina $(\mathrm{N}=134)$ and screened for $Y$. pes tis presence by culture. This period was reported as the peak of the plague epidemic curve, with essentially pneumonic plague cases. Patients had early clinical signs suggesting pulmonary, secondary pulmonary or bubonic plague in an epidemic setting.

The samples containing $K$. pneumoniae were 10 sputum samples and two bubo aspirates and were negative for $Y$. pestis colonies. The description of the 12 patients is shown in (Table 1). Two-thirds of the patients $(\mathrm{N}=8)$ were from Antananarivo and one-third $(\mathrm{N}=4)$ was from Toamasina. Seven patients were men. Five patients were younger than 18 years, and six were 19 to 27 years old and one was 46 years old. Fever status was reported for 10 patients, eight of them had body temperatures $>37.5^{\circ} \mathrm{C}$. According to the clinical forms, nine patients were suspected of having pulmonary plague and one was defined to suffer from secondary pulmonary plague. One patient was suspected of bubonic plague and for another patient, data about the clinical form was lacking.

Four of the patients coughed for at least 5 days and complained of chest pain, although they were in an overall good state of health. Two patients had signs of hemoptysis, and one of 
them was in weak health. Two patients coughed without further complaints but one was in weak health.

Four patients were under antibiotic treatment at the time of sample collection: two with trimethroprim-sulfamethoxazole; one with doxycycline and one with gentamicin. One patient who received trimethroprim-sulfamethoxazole was treated in addition with amoxicillin. There was no mortality among these patients (Table 1).

Abundant colonies with a typical K. pneumoniae morphology (moist, dome-shaped) were recognized after $24 \mathrm{~h}$ culture on CIN medium. The twelve isolates were identified as $K$. pneumoniae by MALDI-TOF. The presence of K. pneumoniae DNA in the two bubo samples was also detected by real time-PCR. Melting curve values for the detection of $K$. pne um oniae were $79^{\circ}$ and $80^{\circ}$ for the positive controls and the DNAs extracted from buboes, respectively (Fig. 2). Of the 12 isolates, four had a positive string test.

Five isolates were ESBL producers. Six isolates were resistant to sulfonamides and trimethoprim. Three and two isolates were resistant to gentamycin and tobramycin, respectively. One isolate was resistant to ciprofloxacin (Table 2).

\section{Genome analys is}

Whole genome sequencing of the $K$. pneumoniae isolates allowed us to characterize cgMLST alleles, virulence genes, capsular loci and resistance genes. All K. pneumoniae isolates were Klebsiella pne umoniae sensu stricto (Kp1) (Fig 1). Among the 12 isolates eight different sequence types (STs) were identified: ST23 (N=1); ST86 (N=2); ST65 (N=1); ST280 $(\mathrm{N}=1)$; ST327 $(\mathrm{N}=1)$; ST380 $(\mathrm{N}=1)$; ST716 $(\mathrm{N}=1)$; and ST3012 $(\mathrm{N}=1)$, and three new STs: ST3441 $(\mathrm{N}=1)$, ST3442 $(\mathrm{N}=1)$, ST3443 $(\mathrm{N}=1)$ (Table 2). Comparative genomic analysis of the two Kp ST86 isolates showed that they differed from each other by 123 alleles out of 632 scgMLST gene loci. 
The virulence genes identified in most isolates were colibactin locus $(c l b)$, siderophores $(\operatorname{Iro} B C D N)$, iron uptake systems and regulators ( $k f u, k v g A$, respectively), yersiniabactin $($ fyuA, irp $1 / 2$ and $y b t)$ (Supplementary data). The PP3-ST380-KL1 isolate, two ST86 isolates and the PP11-ST3443-KL2 isolate were positive in string test. All the ST23, ST65, ST86 and ST380 isolates had the genes $r m p A / r m p A 2$ associated with the hyperproduction of the capsule and also carried the iucABCD genes coding for the synthesis of aerobactin (Supplementary Data).

Isolates belonging to ST23, ST65, ST86 and ST380 were susceptible to all antibiotics tested, with the exception of amoxicillin to which $K$. pne umoniae is intrinsically resistant.

A total of five isolates were ESBL producers (Table 2). A ST280 isolate and a ST3441 isolate carried the bla $a_{\mathrm{CTX}-\mathrm{M}-15}$ gene. A ST3442-KL1 isolate harboring virulence genes $(f y u, i r p 1 / 2, y b t)$ was ESBL producer and carried the cassette comprising qnrB66, aac (3) -IIa, bla SHV-27 $_{\text {and }}$ tet $(\mathrm{A})$, as well as the IncFIB $\mathrm{B}_{\mathrm{K}}$ replicon marker (Table 2).

\section{Discus sion}

Unexpectedly, 12 cases of K. pneumoniae infection were detected and identified among individuals clinically suspected to have plague. No Y. pestis was identified from their clinical samples, but three patients (PP8, PP10 and PP11) yielded a positive result on RDT. However, culture is the gold standard for the identification of $Y$. pes tis and RDT could provide false results ${ }^{16}$. All patients had no epidemiological relationships and no family member or contact had been recorded with plague.

Although the selective medium for Yersinia was not intended for $K$. pne umoniae isolation, $K$. pne um oniae does grow on the CIN medium in $24 \mathrm{~h}$. The selectivity of this medium is reported as being partial, as other Gram-negative bacilli can grow on CIN medium, including other species of Enterobacterales able to ferment mannito ${ }^{17}$. Therefore, full species identification is recommended. 
It is not surprising to isolate $K$. pne umoniae from pneumonia cases. Community-acquired $K$.

220

221

222

223

224

225 pneumoniae infections are common, including in Africa ${ }^{18,19}$. In contrast, to our best knowledge, K. pneumoniae isolated from buboes aspirates were never reported previously. Additionally to culture, the presence of $K$. pneumoniae in buboes aspirates was confirmed by PCR. The advantage of melting curve analysis over Taqman based real time-PCR is its lower costs without losing specificity ${ }^{20}$. Further studies are needed to evaluate this method, which could be used in screening for K. pne um oniae in buboes or other suspected biological samples.

K. pneumoniae has the capacity to acquire resistance genes and to become increasingly more difficult to treat. One of the K. pneumoniae isolates detected in one of two patients who was treated by combination of amoxicillin and trimethoprim-sulfamethoxazole developed resistance. However, K. pne umoniae is known to be intrinsically resistant to ampicillin due to the presence of the chromosomal B-lactamase SHV-1 or similar ${ }^{21}$. At the same time, we identified in this strain (ST280), the two genes $d f r A 14$ and $s u l 2$, associated to resistance to trimethoprim and sulfamethoxazole, respectively. In addition, among all isolates, it was the only $K$. pneumoniae isolates to be resistant to piperacillin/tazobactam (TZP), which is concordant with the presence of bla TEM-1B gene $^{22}$.

Two of the five ESBL isolates harbored bla $a_{\mathrm{CTX}-\mathrm{M} 15}$. This gene was commonly found in ESBLproducing K. pne umoniae isolated in Madagascar ${ }^{19}$. bla $_{\mathrm{SHV} 27}$ and bla $_{\mathrm{SHV} 101}$ were found in the other ESBL-producer.

According to MLST analysis, we observed the presence of five STs known to be associated with hypervirulence, including STs ST23 $(\mathrm{N}=1)$, ST65 $(\mathrm{N}=1)$, ST86 $(\mathrm{N}=2)$ and ST380 $(\mathrm{N}=1)$. In addition, a K. pne umoniae isolate having the new ST3443 differs by a single locus from ST86 on the tonB locus (allele 18 instead of 27). This strain ST3443 tested string positive, as was also the case for the ST380 and two ST86 strains. Whole genome analysis of $K$. pne umoniae showed that the isolates with common ST differed to each other by alleles 
occurring outside the 7 household genes. The presence of common virulence genes in the ST23 isolate which were ICEKp10 encoding $c l b 2$ sequence variants, ybt 1 and $r m p A / r m p A 2$ suggests its belonging to CG23 sublineage I (CG23-I) ${ }^{23}$. MLST typing of $K$. pneumoniae isolated in different countries revealed that ST23, ST65, ST86 and ST380 were responsible for hepatic abscess cases and other invasive CA infections $^{24}$.These isolates have been reported particularly in Asia, but their diffusion outside Asia has been described ${ }^{24}$. Among virulence factors, $m p A$ and aerobactin are the most important one ${ }^{25}$. The presence of genes responsible for the hypermucoviscosity phenotype, $r m p A / r m p A 2$, plays an important role in the virulence of $K$. pneumoniae isolates. These genes are often associated with serotype K1 and K2. Expression of these genes allows the bacteria to escape the host's defense system and colonize the mucous membranes. Epidemiological studies have shown that the majority of ST23 are related to K1 capsular serotypes and liver abscesses ${ }^{23,26}$, while $\mathrm{K} 2$ is the second capsular serotype resulting in community-acquired pneumonia ${ }^{25}$. Yersiniabactin, a virulence gene $(Y b t)$, detected in the three K. pneumoniae isolates serotyped $\mathrm{K} 1$ has been reported as the iron absorption system in highly virulent $Y$. pestis ${ }^{27}$, but was shown to have evolved ancestrally within the Kle bs ie lla genus $^{23}$. Several studies have shown isolates belonging to these STs $(23,65,86$ and 380$)$, with the same combination of virulence factors, to be virulent in mouse models ${ }^{12,23}$. During an epidemic, knowledge of the etiology is essential in order to provide the most adapted treatment to patients. Microbiological diagnosis can improve the effectiveness of treatments, avoid long-term complications for the infected patient, and in addition avoid widespread overuse and misuse of antibiotics. Early diagnosis can help to prevent or stop an outbreak too. One of the reasons for a possible treatment failure could arise during inaccurate diagnoses and inappropriate treatments. Similar symptoms can lead to routine treatments based on syndromic approaches which are often applied in developing countries. As the physician is rarely able to make an etiological diagnosis on clinical grounds alone, 
271 treatment should ideally be based on the result of bacteriological examination. In this case, 272 bacteriological diagnosis could be complicated by the fact that the respiratory tract could be 273 infected by K. pneumoniae ${ }^{28}$. Although the population we included in our study is young, the

274 clinical signs of a few patients warned us of possible serious infections due to K. pne umoniae 275 such as bloody sputum and a chest pain which were among typical signs of pestis pneumonia, however, $Y$. pestis was not found in culture. Typically, the plague is better known 277 by its three clinical forms: bubonic, septicemic and pulmonary plague while hypervirulent $K$. 278 pneumoniae strains are known to cause pneumonia, sepsis, liver abscesses and 279 meningitis $29-31$.

280 We acknowledge the following limitations of our study. First, we studied a limited number of samples in a short duration of the epidemic, which is far from being representative of all the negative samples for $Y$. pestis. Second, detailed data about clinical characteristics and outcomes were lacking due to the outbreak emergency context. Finally, we did not confirm 284 the virulence of the K. pne umoniae strains using mouse models.

\section{Conclusions}

Although few samples were studied, within a short duration of inclusion (9 days from 06/10/17 to 14/10/17), our results show that the plague-suspected patients in fact acquired a pneumonia caused by K. pneumoniae. Bacterial identification proved useful for determining 290 the etiology. WGS and AST results showed that among the $12 \mathrm{~K}$. pne umoniae isolates, there 291 were ESBL producers and virulent strains. This study shows that during epidemics of plague, clinicians and microbiologists should also consider other pathogens, especially $K$. pne umoniae given the fact that the clinical symptoms of respiratory plague can be confused with other pathologies. 


\section{Funding}

298 Internal funding.

299

300

\section{Competing interests}

301 None.

302

303

\section{Ethical approval}

304 Not required.

305

306

Acknowledgements

307

We are deeply grateful to the curators of the Institut Pasteur MLST system (Paris, France) for importing novel alleles, profiles isolates at https://bigsdb.pasteur.fr.

309

310

\section{Author contributions}

A.R., T.C., V.J., S.B., M.R. and J.C. wrote the manuscript. A.R., L.M.A., S.A.R., S.H.R., V.A.,

S.R. performed lab experiments. S.A.R, V.A., S.R., M.R completed data collection. A.R., S.B., V.J., M.R. and J.C. conceived the study. A.R., T.C., S.B., M.R. and J.C. analysed and interpreted data. All authors read and approved the final manuscript.

\section{References}

1. Randremanana, R., Andrianaivoarimanana, V., Nikolay, B. et al. Epidemiological characteristics of an urban plague epidemic in Madagascar, August-November, 2017: an outbreak report. Lancet Infect Dis 19, 537-545 (2019).

2. WHO. International meeting on preventing and controlling plague: the old calamity still 
has a future. Wkly Epidem iol Rec 81, 278-84 (2006).

324

325

326

327

328

329

330

331

332

333

334

335

336

3. Andrianaivoarimanana, V., Piola, P., Wagner, D.M., et al. Trends of Human Plague, Madagascar, 1998-2016. Emerg Infect Dis . 25(2):220-228 (2019).

4. Rodrigues, C., Passet, V., Rakotondrasoa, A. Diallo, T.A., Criscuolo, A., Brisse, S. Description of Kle bs ie lla africanens is sp. nov., Kle bs ie lla variic ola subsp. tropicalens is subsp. nov. and Kle bs ie lla variicola subsp. variicola subsp. nov. Res Microbiol. 170: 165-170 (2019). 5. Wyres, K.L., Holt, K.E. Klebsiella pneumoniae as a key trafficker of drug resistance genes from environmental to clinically important bacteria. Curr Opin Microbiol. 45, 131-139 (2018).

6. Mizuta, K., Ohta, M., Mori, M., Hasegawa, T., Nakashima, I., Kato, N. Virulence for mice of Klebsiella strains belonging to the $\mathrm{O}$ group: relationship to their capsular $(\mathrm{K})$ types. Infect Imm un. 40(1):56-61 (1983).

7. Cortés, G., Borrell, N., de Astorza, B., Gómez, C., Sauleda, J., Albertí, S. Molecular analysis of the contribution of the capsular polysaccharide and the lipopolysaccharide $O$ side chain to the virulence of Klebsiella pneumoniae in a murine model of pneumonia. Infect Immun. 70(5):2583-90 (2002).

8. Tomás, J.M., Camprubi, S., Merino, S., Davey, M.R., Williams, P. Surface exposure of $\mathrm{O} 1$ serotype lipopolysaccharide in Klebsiella pneumoniae strains expressing different $\mathrm{K}$ antigens. Infect Im mun. 59(6):2006-11 (1991).

9. Di Martino, P., Cafferini, N., Joly, B., Darfeuille-Michaud, A. Klebsiella pneumoniae type 3 pili facilitate adherence and biofilm formation on abiotic surfaces. Res Microbiol. 154(1):9-16 (2003).

10. Turton, J. F., Baklan, H., Siu, L. K., Kaufmann, M. E., Pitt, T. L. Evaluation of a multiplex PCR for detection of serotypes $\mathrm{K} 1, \mathrm{~K} 2$ and $\mathrm{K} 5$ in Klebsiella sp. and comparison of isolates within these serotypes. FEMS Microbiol. Lett. 284, 247-252 (2008).

11. Lin, W. H. et al. Clinical and microbiological characteristics of Klebsiella pneumoniae 
isolates causing community-acquired urinary tract infections. Infection 38, 459-464 (2010).

12. Shon, A.S., Bajwa, R.P., Russo, T. A. Hypervirulent (hypermucoviscous) Klebsiella pneumoniae: a new and dangerous breed. Virulence . 4(2):107-18 (2013).

13. WHO. Interregional meeting on prevention and control of plague. Geneva: World Health Organization, 2008.

14. Passet, V. \& Brisse, S. Association of tellurite resistance with hypervirulent clonal groups of Klebsiella pneumoniae. J. Clin. Microbiol. 53, 1380-1382 (2015).

15. Barbier, E., Rodrigues, C., Depret, G., et al. The ZKIR Assay, a Real-Time PCR Method for the Detection of Klebsiella pneumoniae and Closely Related Species in Environmental Samples. Appl Environ Microbiol. 86(7):e02711-19 (2020).

16. Rajerison, M., Melocco, M., Andrianaivoarimanana, V., Rahajandraibe, S., Rakotoarimanana, F., et al. Performance of plague rapid diagnostic test compared to bacteriology; a retrospective analysis of the data collected in Madagascar. BMC Infectious dise ases . $20(90)(2020)$.

17. Weagant, S.D. A new chromogenic agar medium for detection of potentially virulent Yersinia enterocolitica. J Microbiol Methods. 2008 Feb;72(2):185-90. doi: 10.1016/j.mimet.2007.11.019. Epub 2007 Dec 4. PMID: 18177960.

18. Huynh, B.T., Passet, V., Rakotondrasoa, A., Diallo, T., Kerleguer, A., et al. Kle bs ie lla pne umoniae carriage in low-income countries: antimicrobial resistance, genomic diversity and risk factors. Gut Microbes . 2020;11(5):1287-1299 (2020).

19. Rakotondrasoa, A., Passet, V., Herindrainy, P., Garin, B., Kermorvant-Duchemin, E., et al. Characterization of Klebsiella pneumoniae isolates from a mother-child cohort in Madagascar. J Antim ic rob Chemother. 75(7):1736-1746 (2020).

20. Tajadini, M., Panjehpour, M., Javanmard, S.H. Comparison of SYBR Green and TaqMan methods in quantitative real-time polymerase chain reaction analysis of four adenosine receptor subtypes. Adv Biomed Res. 2014 Feb 28;3:85. doi: 10.4103/2277- 
9175.127998. PMID: 24761393; PMCID: PMC3988599.

21. Fu, Y., Zhang, F., Zhang, W., Chen, X., Zhao, Y., et al. Differential expression of bla(SHV) related to susceptibility to ampicillin in Klebsiella pneumoniae. Int J Antimicrob Agents . 29(3):344-7 (2007).

22. Hubbard, A.T.M., Mason, J., Roberts, P., Parry, C.M., Corless, C., et al. Piperacillin/tazobactam resistance in a clinical isolate of Escherichia coli due to IS26mediated amplification of bla

23. Lam, M.M.C., Wyres, K.L., Duchêne, S., Wick, R.R., Judd, L.M., et al. Population genomics of hypervirulent Klebsiella pneumoniae clonal-group 23 reveals early emergence and rapid global dissemination. Nat Com mun. 9(1):2703 (2018).

24. Diancourt, L., Passet, V., Verhoef, J., Grimont, P. A. D. \& Brisse, S. Multilocus sequence typing of Klebsiella pneumoniae nosocomial isolates multilocus sequence typing of Klebsiella pneumoniae nosocomial isolates. J. Clin. Microbiol. 43, 4178-4182 (2005).

25. Holt, K.E., Wertheim, H., Zadoks, R.N., Baker, S., Whitehouse, C.A., et al. Genomic analysis of diversity, population structure, virulence, and antimicrobial resistance in Klebsiella pneumoniae, an urgent threat to public health. Proc Natl Acad Sci. 112(27):E3574-81 (2015). 26. Liao, C.H., Huang, Y.T., Chang, C.Y., Hsu, H.S., Hsueh, P.R. Capsular serotypes and multilocus sequence types of bacteremic Klebsiella pneumoniae isolates associated with different types of infections. Eur J Clin Microbiol Infect Dis . 33(3):365-9 (2014).

27. Rakin, A., Schneider, L., Podladchikova, O. Hunger for iron: the alternative siderophore iron scavenging systems in highly virulent Yersinia. Front Cell Infect Microbiol. 2:151 (2012)

28. Sasaki, E., Tokiwa, T., Tsugo, K., Higashi, Y., Hori, H., et al. Peracute Bacterial Meningitis due to Infection with Klebsiella pneumoniae in Captive-bred Ruffed Lemurs (Varecia variegate). J Comp Pathol. 156(2-3):281-285 (2017).

29. Galy, A., Loubet, P., Peiffer-Smadja, N., Yazdanpanah, Y. The plague: An overview 
401 and hot topics. Rev Med Interne. 39(11):863-868 (2018).

402 30. Fung, C., Chang, F., Lee, S., Hu, B.S., Kuo, B.I. et al. A global emerging disease of 403 Klebsiella pneumoniae liver abscess? is serotype $\mathrm{K} 1$ an important factor for complicated 404 endophthalmitis. Gut. 50: 420-4 (2002).

405 31. Chung, D.R., Lee, H., Park, M.H., Jung, S.I., Chang, H.H. et al. Fecal carriage of 406 serotype K1 Klebsie lla pne umoniae ST23 strains closely related to liver abscess isolates in 407 Koreans living in Korea. Eur J Clin Microbiol Infect Dis . 31: 481-6 (2012). 
Figures

Tree scale: $0.01 \longmapsto$

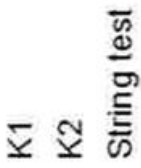

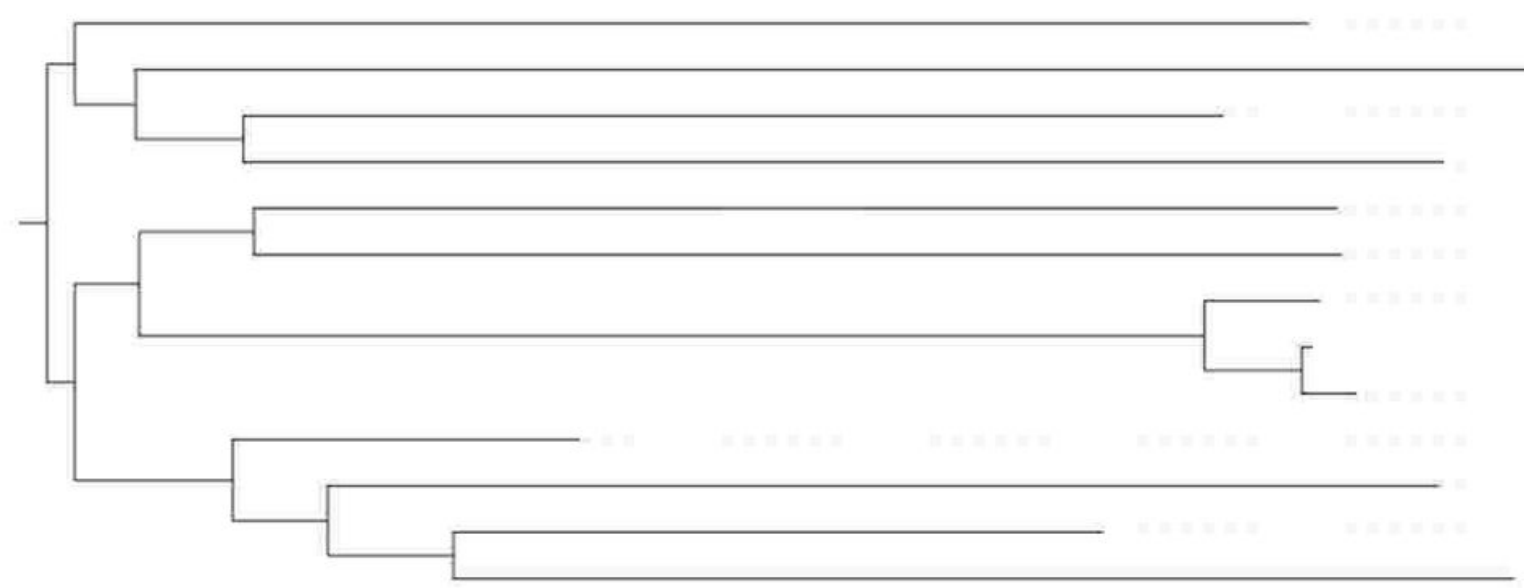

Kp1

PP10 ST65

PP4 ST3442

PP7 ST3012

PP8 $\quad$ ST716

PP1 ST280

PP12 ST86

PP9 ST86

PP11 ST3443

PP2 ST3441

PP5 ST23

PP3 $\mathrm{ST} 380$

PP6 $\quad$ ST327

Figure 1

Phylogenetic tree of $12 \mathrm{~K}$. pneumoniae isolates

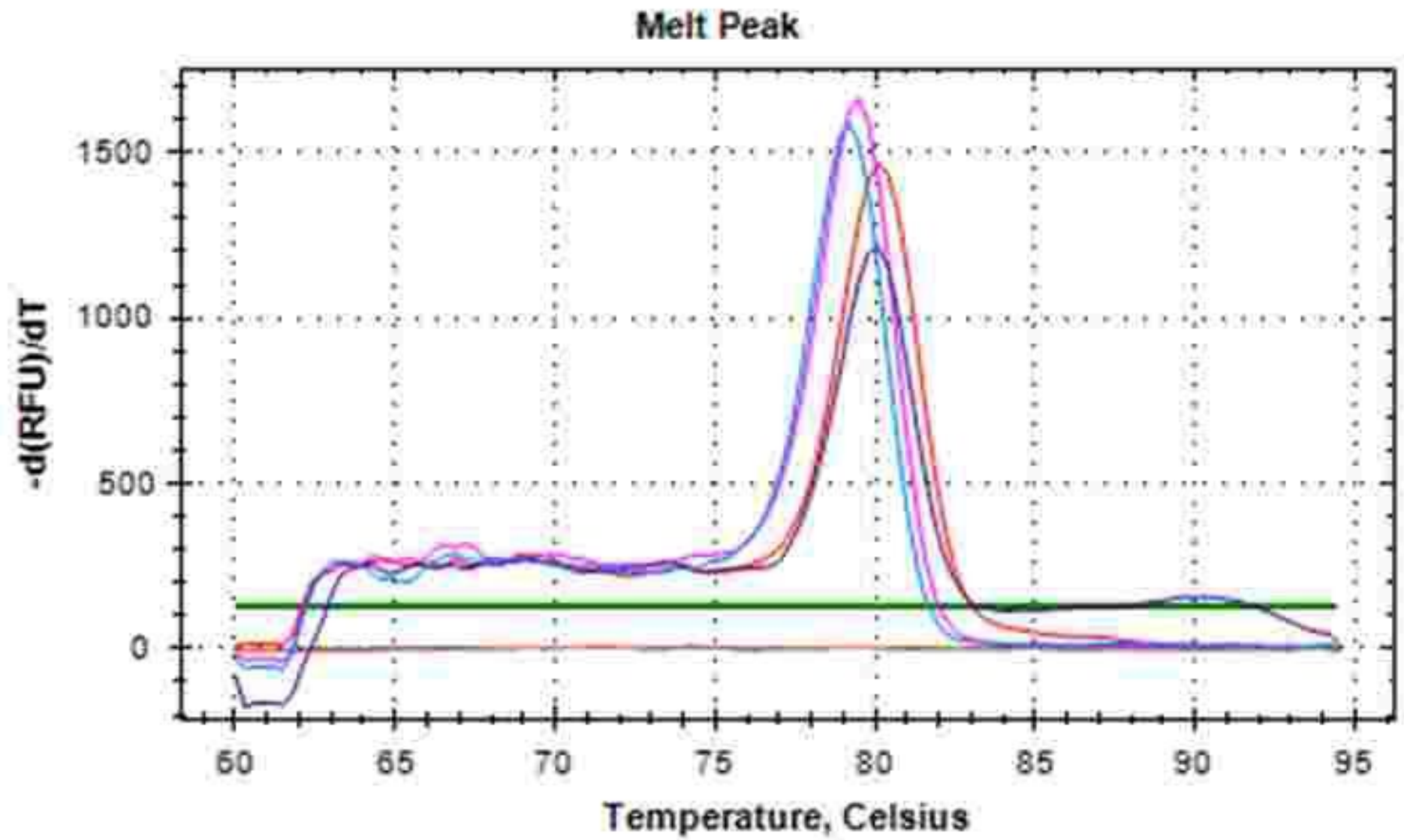

Figure 2 
Melting curve results from ZKIR region detection obtained after 40 cycles on the ZKIR quantitative PCR system of Kp. DNA from two positive controls from strains blue to UUA 2239, violet to UUA 2016. DNA extracted from patients' buboes in pink to PP2 and red color to PP8. Orange color corresponds to negative control.

\section{Supplementary Files}

This is a list of supplementary files associated with this preprint. Click to download.

- Table.pdf

- Tables1.csv 